\title{
X-RAY ABSORPTION SPECTROSCOPY
}

\author{
S. Moвilio \\ Dipartimento di Energetica Universita' de L'Aquila \\ Roio Monteluco 67100 L' Aquila, Italy \\ and \\ Laboratori Nazionali di Frascati \\ via E. Fermi 40, 00044 Frascati, Italy
}

\begin{abstract}
X-ray absorption spectroscopy, today widely used as a tool for structural investigations, is described. Theoretically it is shown that above an absorption edge the absorption coefficient is well approximated by a sum of terms, each one describing a particular path followed by the photoelectron outgoing from the atom. Such geometrical feature allows to use X-ray absorption spectroscopy for structural investigations. The data analysis procedures are outlined. Applications of the single scattering region, generally known as EXAFS to semiconductors alloys, to metallic clusters and to the crystallization of amorphous metallic glasses are illustrated. Multiple scattering calculations and their relation to experimental spectra is discussed in the case of $\mathrm{Mn}$ ions in solution. Application of the multiple scattering to determine the three-body correlation function in a-Si:H is shown.
\end{abstract}

PACS numbers: 78.70.Dm, 07.85.+n

\section{Introduction}

X-ray absorption spectra (XAS) have been discovered and extensively investigated during the first half of this century. The development of the quantum mechanics explained that the excitation of inner shell electrons to free bound states or to the continuum gives rise to absorption edges in the X-ray region. Since that time, at photon energies above the edge fine structures were observed. Such fine structures, extending up to hundreds eV above the edge are not observed in monoatornic systems, but only in polyatomic compounds. Even if it was soon clear that the fine structures reflect the details of the final states wave functions, only two decades ago an approximate, but enough accurate theory of the X-ray absorption coefficient and of its fine structures was developed [1, 2]: that time it was shown that one can get from X-ray spectra the geometrical arrangement of the atoms around the one, whose $\mathrm{X}$-ray absorption edge is measured [3]. In the meantime, the development of synchrotron radiation facilities, which provide high resolution and high flux X-ray beams, allowed to record high quality XAS 
spectra in few minutes, instead of the days needed with an X-ray tube so giving a great impetus to the application of X-ray absorption spectroscopy. Thanks to these two events, today the X-ray absorption spectroscopy is extensively used as a local structural probe in widespread fields of physics, chemistry, biology, material and surface science [4-6].

In the present lecture the rnodern interpretation of the X-ray absorption spectra in terms of multiple scattering (MS) formalism is outlined, showing that above a certain photon energy the absorption coefficient is given by a sum of terms, each one describing a scattering path followed by the photoelectron in living the absorbing atom. It is this geometrical aspect which allows to get structural information from the spectra. Emphasis will be given to the description of the dominant term, the second in the expansion, today known as extended X-ray absorption fine structure (EXAFS). Typical applications of EXAFS will be illustrated. The presence of sizeable multiple-scattering contribution in XAS spectra will be shown.

)

\section{Origin of X-ray absorption fine structures}

It is usual to divide X-ray absorption spectra in three regions [7] (Fig. 1):

1) the "edge region"; which spans roughly $10 \mathrm{eV}$ above and below the edge;

2) the "X-ray absorption near edge structure" region, extending from the edge up to $30 \mathrm{eV}$ above it, and known as XANES or NES or NEXAFS;

3 ) the "extended X-ray absorption fine structure" (EXAFS) region extending from about $30 \mathrm{eV}$ above the edge up to even $1000 \mathrm{eV}$.

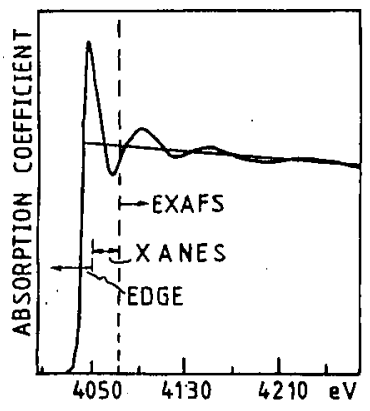

Fig. 1. X-ray absorption spectrum of a $\mathrm{Ca}$ compound around the $\mathrm{Ca} K$ edge. The different spectral regions are indicated.

In the edge region the absorption coefficient resembles the site projected density of final states, providing information on the electronic structure. In the XANES and EXAFS regions, between which no formal separation exists, the absorption coefficient is connected to the structure around the absorbing center.

To justify such classification it is necessary to look at the theory of the absorption cross-section. As known in the dipole approximation the absorption cross-section $\sigma$ is given by

$$
\sigma(h \omega)=4 \pi^{2} \alpha h \omega \sum_{f}\left|\left\langle\Psi_{\mathrm{fin}}|\varepsilon p| \Psi_{\mathrm{in}}\right\rangle\right|^{2} \delta\left(E-E_{\mathrm{f}}+E_{\mathrm{i}}\right) \quad\left(\alpha=e^{2} / h c\right),
$$


where $h \omega$ is the photon energy, $\varepsilon$ - its polarization vector, $p$ - the dipole transition operator. The initial state wave function $\Psi_{\text {in }}$ of an inner shell electron is generally well described by atomic wave functions so can be considered as known. The problem is the calculation of the photoelectron final state $\Psi_{\text {fin }}$.

Let us start first with an isolated atom. In a very simple picture the final state photoelectron wave function is well described by a free spherical wave, outgoing from the atom. In such a case no fine structure is predicted unless in the edge region in agreement with the experimental observations. If the atom is in a matrix, the free wave outgoing from the absorber feels the potential of the surrounding atoms, so being distorted. The distortion can be described as a scattering of the outgoing wave by the potential of the atoms surrounding the absorbing one. The final state wave function becomes the superposition of the free outgoing wave and the scattered one. Such scattered wave can be scattered again by another atom giving rise to a new wave and so on. The interference between all waves produces minima and maxima in space. Since the initial state is very localized in the nearby of the origin, from the cross-section (1) minima and maxima are expected in the spectra when a minimum or a maximum is present at the origin. This will happen at energy values depending on the photoelectron wave vector $k$, on the geornetry of the systern around the absorbing atorn, which determines the phase difference between the interfering waves and on the scattering characteristics of the surrounding atoms. In such picture, the EXAFS region is characterized by a final state built up by the superposition of the primary outgoing wave and the waves scattered only once by a surrounding atom: therefore it carries information only on the distance $R_{j}$ between the absorber and the scatterer (Fig. 2a). The

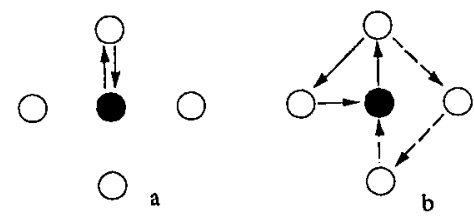

Fig. 2. Scattering paths followed by the photoelectron: (a) EXAFS-like scattering; (b) triple (-) and quadruple (- -) scattering paths.

XANES region is characterized by the presence of triple, quadruple and higher scattering contributions and carries information on the relative arrangement of the atoms around the center, namely on the angles between bonds (Fig. 2b). The edge region finally is determined by all possible scattering at any order: to be more precise in the edge region the procedure of building up the final state by adding scattering paths does not converge. In such region the structural information is hindered and only particular structural characteristics of the final state can be discovered, like the symmetry.

Theory shows that in the multiple scattering scheme the absorption cross-section can be factorized as

$$
\sigma(h \omega)=\sigma_{0}(h \omega) \chi(h \omega)
$$


where $\sigma_{0}(h \omega)$ is the atomic cross-section and $\chi(h \omega)$ is a structural factor, for a $K$ shell given by

$$
\chi=1 /\left(3 \sin ^{2} \delta_{01}\right) \sum_{m} \operatorname{Im}\left\{\left[\left(I-T_{A} G_{0}\right)^{-1} T_{A}\right]_{(1 m, 1 m)}^{0,0}\right\} .
$$

In this expression $\delta_{01}$ is the phase shift of the central atom potential for a spherical wave of $l=1$ angular momentum, $G_{0}$ is the free propagator and $T_{A}$ is the $T$-matrix of the electronic potential of the system. In the simplest approximation, good only for fixing idea, $T_{A}$ is the superposition of the $t$-matrix of the electronic potential of the single isolated atoms.

The problem of calculating the XAS spectra is reduced to the calculation of the matrix elements of the operator $\left(I-T_{A} G_{0}\right)^{-1} T_{A}$, between an outgoing spherical wave of angular momentum $(1 \mathrm{~m})$ and an ingoing spherical wave with the same angular momentum. The energy region where it is necessary to resolve the full Eq. (3) to reproduce the spectra is the "full multiple scattering region" or "edge region", where the connection between the absorption coefficient and the structure is very indirect. The situation changes at high enough energy above the edge, when becomes possible to expand the operator $\left(I-T_{A} G_{0}\right)^{-1} T_{A}$ in series of $T_{A} G_{0}$. Since in this case

$$
\left(I-T_{A} G_{0}\right)^{-1}=\sum_{n}(-1)^{n}\left(T_{A} G_{0}\right)^{n}
$$

we get

$$
\sigma=\sigma_{0} \sum_{n} \chi_{n}
$$

with

$$
\chi_{n}=(-1)^{n} /\left(3 \sin ^{2} \delta_{01}\right) \sum_{n} \operatorname{Im}\left\{\left[\left(T_{A} G_{0}\right)^{n} T_{A}\right]_{1 m, 1 m}^{0,0}\right\} .
$$

Mathernatically the series expansion can be performed only when the moduli of all the eigenvalues of the operator $T_{A} G_{0}$ are smaller than one. Practically this becomes possible only at high photoelectron kinetic energies. It is not possible to give in general an energy value above which the expansion is viable: in the case of $\mathrm{Cu}$ the series converges few $\mathrm{eV}$ above the edge, while in other cases, like Si, more than $30 \mathrm{eV}$ are needed. The meaning of each $\chi_{n}$ is the following: $\chi_{0}$ is identically $1-$ it means that at zero order the spectrum is that of an isolated atom. $\chi_{1}$ is identically zero, since the operator $G$ is off diagonal in the site indices. $\chi_{2}$ is the EXAFS: it describes a spherical wave coming out from the absorber which propagates toward another atom, scatters from it, then propagates again toward the absorber and is scattered again from the absorber, before reaching the origin. $\chi_{3}$ describes the triple scattering and so on. It turns out that at any energy, apart the atomic term, the EXAFS term $\chi_{2}$ is the dominant one and describes the main features of the fine structures present in the spectrum. The multiple scattering contribution $\chi_{n}$ for $n>3$ are generally hindered by the EXAFS: anyway, using accurate data analysis, their contribution is detected in many cases. In first instance we will neglect their presence in the XAS spectra and assume that the only information present in the spectra is the EXAFS term. 


\section{Extended X-ray absorption fine structure}

\subsection{Theory}

According to the expansion (6), and assuming no significant multiple scattering contribution, the XAS spectrum $\chi_{2}$ is given by

$$
\chi_{2}=\left(\sigma-\sigma_{0}\right) / \sigma_{0}=1 /\left(3 \sin ^{2} \delta_{01}\right) \sum_{m} \operatorname{Im}\left\{\left[T_{A} G_{0} T_{A} G_{0} T_{A}\right]_{(1 m, 1 m)}^{0,0}\right\} .
$$

Since the effect of $T_{A}$ at the origin, where only the potential due to the central atom is present, is to multiply the spherical wave by $t_{1}=\mathrm{e}^{\mathrm{i} \delta_{01}} \sin \delta_{01}$ where $\delta_{01}$ is the corresponding phase shift, it turns out that [1]

$$
\chi_{2}=\operatorname{Im}\left[1 / 3 \mathrm{e}^{\mathrm{i} 2 \delta_{01}} \sum_{m} \sum_{j} G_{\left(l m, l^{\prime} m^{\prime}\right)}^{0, j} t_{l^{\prime}}^{j} G_{\left({ }^{\prime} m^{\prime}, l m\right)}^{j, 0}\right] .
$$

Introducing the explicit expression for the propagators $G_{\left(l^{\prime} m^{\prime}, l m\right)}^{j, i}$ and remembering again that the effect of $t_{l}^{j}$ is to multiply the wave function by $\mathrm{e}^{\mathrm{i} \delta_{j l}} \sin \delta_{j l}$ where now $\delta_{j l}$ is the phase shift of the atom at $R_{j}$ we get

$$
\chi_{2}=\operatorname{Im}\left[\mathrm{e}^{\mathrm{i} 2 \delta_{01}} \sum_{j} \sum_{l^{\prime}}\left(2 l^{\prime}+1\right) \mathrm{e}^{\mathrm{i} \delta_{j l^{\prime}}} \sin \left(\delta_{j} l^{\prime}\right) H\left(1, l^{\prime} ; R_{j}\right)\right]
$$

with

$$
H\left(1, l^{\prime} ; R_{j}\right)=\sum_{l^{\prime \prime}}\left(2 l^{\prime \prime}+1\right)\left[\left\{1 l^{\prime} l^{\prime \prime} ; 000\right\} h_{l^{\prime \prime}}^{+}\left(k R_{j}\right)\right]^{2},
$$

$\left\{1 l^{\prime} l^{\prime \prime} ; 000\right\}$ being the Wigner $3 j$ symbols. This is the exact EXAFS formula, generally named spherical wave EXAFS [8].

The most used approximation to formula (10) is the so-called "plane wave approximation", valid for $K R_{j} \gg 1$, which corresponds to approximate the Hankel function $h_{l^{\prime \prime}}^{+}\left(k R_{j}\right)$ on each scatterer with a plane wave $\mathrm{e}^{i k R_{j}} / k R_{j}$. With this approximation, it turns out that

$$
H\left(1, l^{\prime} ; R_{j}\right)=(-1)^{1+l^{\prime}} \mathrm{e}^{\mathrm{i} 2 k R_{i}} /\left(k R_{j}\right)^{2}
$$

and

$$
\chi_{2}=-\sum_{j} \operatorname{Im}\left\{\mathrm{e}^{\mathrm{i} 2 \delta_{0 l}} \sum_{l^{\prime}}\left(2 l^{\prime}+1\right) \mathrm{e}^{\mathrm{i} \delta_{j l^{\prime}}} \sin \left(\delta_{j l}\right)(-1)^{i^{\prime}} \mathrm{e}^{\mathrm{i} 2 k R_{j}} /\left(k R_{j}\right)^{2}\right\} .
$$

Since the scattering amplitude in the back direction $f_{j}(k, \pi)$ of the atomic potential at $R_{j}$ is connected to the phase shift through

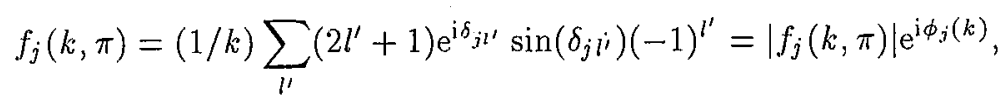

we finally get.

$$
\chi_{2}=-\sum_{j}\left|f_{j}(k, \pi)\right| /\left(k R_{j}^{2}\right) \sin \left(2 k R_{j}+\phi_{j}(k)+2 \delta_{01}\right) .
$$


This formula tells that each atom at a distance $R_{i j}$ from the absorber contributes to the spectrum with a pure sine-like oscillation of frequency $2 R_{j}$. The frequency and the phase of the oscillation are modified by two phase terms $\phi_{j}(k)$ and $2 \delta_{01}$ arising the first from the scattering of the photoelectron, assumed as a plane wave, in the field of the backscattering atom, the second from the phase shift suffered by the photoelectron in passing twice through the central atom potential. The $k$ behavior of the oscillations is governed by the function $\left|f_{j}(k, \pi)\right|$ which is strongly dependent on the $Z$ number of the backscattering atoms. Other factors not yet considered, which modify the amplitude of the oscillations are: the thermal fluctuations of the distances $R_{i j}$, which in the harmonic approximation are well accounted for by an additional Debye-Waller like factor $\exp \left(-2 k^{2} \sigma^{2}\right)$; the lifetime of both the core hole and the photoelectron which are accounted for by an exponential-like term $\exp \left(-2 R_{j} / \lambda\right)$, where $\lambda(k)$ is the mean free path of the photoelectron in the excited state, due to the two finite lifetimes; the presence of multielectron effect which are approximated by a constant reduction factor $S_{0}^{2}$, whose value is always smaller than 1 .

In conclusion, taking into account also these effects, the plane wave EXAFS is described by the following formula:

$$
\begin{aligned}
\chi_{2}= & -\sum_{j} N_{j}\left|f_{j}(k, \pi)\right| /\left(k R_{j}^{2}\right) \exp \left(-2 k^{2} \sigma^{2}\right) \exp \left(-2 R_{j} / \lambda\right) \\
& \times \sin \left[2 k R_{j}+\phi_{j}(k)+2 \delta_{01}\right],
\end{aligned}
$$

where the sum over the single atoms has been substituted by the surn over the coordination shells around the absorber, each one corrosed by $N_{j}$ atoms.

Looking at this expression we easily discover that the structural information gained from an EXAFS spectrum is local: the factors $1 / k R_{j}^{2}$ and $\exp \left(-2 R_{j} / \lambda\right)$ reduce the contribution of the coordination shells far from the absorber: typically quantitative structural data are gained up to the third-fourth coordination shell, in lucky cases.

\subsection{Data analysis}

The data analysis of the spectra requires first the evaluation from the experimental spectra of the structural part $\chi=\left(\sigma-\sigma_{0}\right) / \sigma_{0}$ and then the analysis of $\chi$ in terms of the EXAFS formulas. Since $\sigma_{0}$ generally is not known with enough accuracy, it is determined numerically from the absorption spectrum, by fitting the absorption coefficient above the edge by a smooth polynomial spline, which, passing through the average of the structural oscillations, reproduces $\sigma_{0}$. A second step is the conversion of the energy scale to the photoelectron wave vector scale. If $E_{0}$ is the ionization energy of the inner shell electron, then obviously

$$
k=\left(2 m / h^{2}\right)^{1 / 2}\left(h \omega-E_{0}\right)^{1 / 2} .
$$

The exact knowledge of $E_{0}$ is very difficult: there is no way to connect a particular feature of the edge, to the ionization energy $E_{0}$. It is custornary to assume $E_{0}$ at the inflection point of the edge, since in the atornic case if the core electron could be excited directly into the continuum, the edge line shape should be

$$
\alpha(E)=\alpha(\infty)\left\{1 / 2+(1 / \pi) \arctan \left[2\left(E-E_{0}\right) / \Gamma_{i}\right]\right\}
$$


where $\Gamma_{i}$ is the core level width. Anyhow, even in the pure atomic case such choice is arbitrary since also excitations to bound states are present. From the EXAFS point of view, such uncertainty deforms the $k$ scale, worsening the accuracy of distance determinations. Anyway, procedures exist which, requiring self-consistency inside the data analysis, allow to improve the $E_{0}$ choice, so minimizing the error in distance determinations.

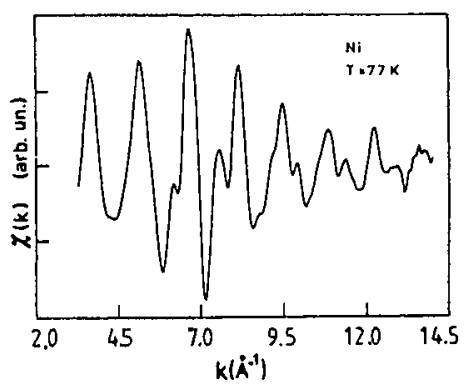

Fig. 3. EXAFS spectrum of a Ni metallic foil at liquid nitrogen.

As a result of the previous two steps of data analysis, one obtains from a XAS spectrum the EXAFS spectra (Fig. 3). The determination of the structural parameters $R_{j}, N_{j}$, and $\sigma_{j}^{2}$ for each coordination shell contributing to the signal, requires the knowledge of the scattering functions $\left|f_{j}(k, \pi)\right|, \phi_{j}(k)$ and $\delta_{01}(k)$ and of the photoelectron mean free path. At present there exist reliable published theoretical values $[9,10]$ of the phase functions $\phi_{j}(k)$ and $\delta_{01}(k)$ and refined computer codes to evaluate these functions, so they can be considered as well known. As for $\left|f_{j}(k, \pi)\right|$ the reliability of the calculations is somewhat smaller [11], since this function is much more sensitive to the details of the potential: approximations like the muffin-tin potentials can be too crude. Moreover, the mean free path damping and the many body correction affect the total amplitude of the EXAFS oscillations. Anyhow with an indetermination of $10 \%$, also the amplitude factors can be considered as known. As a consequence the structural parameters $R_{i j}, N_{j}$, and $\sigma_{j}^{2}$ can be determined with an accuracy of $0.02 \AA, 10 \%$ and $10 \%$ respectively. At present, a big theoretical effort exists in developing ab initio calculations of EXAFS, using complex potential. Such calculations are able to reproduce scattering phase and amplitudes as well as inelastic losses, but today they are not yet able to increase the accuracy of the structural determinations; they however take into account the correction due to curved wave effects $[10,12]$.

An up to now better approach is to use the so-called phase [4] and amplitude [10] transferability, which states that, analyzing a particular EXAFS contribution to a spectrum, the total phase function $\left[\phi_{j}(k)+2 \delta_{0 l}(k)\right]$ and the total armplitude function $\left[\left|f_{j}(k, \pi)\right| \exp \left(-2 R_{j} / \lambda\right)\right]$ can be determined studying a model system whose structural properties are known, and then used to analyze the spectrum in an unknown, provided the two compounds are chernically similar. Using such a method accuracy as high as $0.005 \AA$ and $1 \%$ in distance and coordination number determinations have been reported in literature. 
FOURIER TRANSFORM

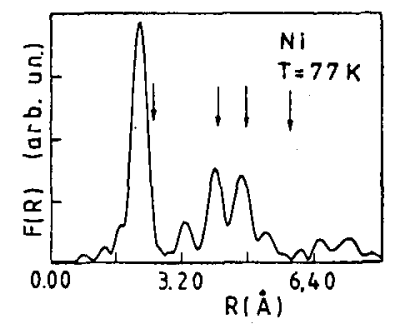

Fig. 4. Fourier transform of the EXAFS spectrum for metallic Ni. Arrows show the crystallographic positions; the shift is due to the scattering phase.

It is obvious from Eq. (15), that the Fourier transform (FT) of the EXAFS will present a peak for each coordination shell, whose maximurn $R_{\max }$, properly corrected, gives the $R_{j}$ value for that shell (Fig. 4). The correction, needed for the presence of the total phase $\left[\phi_{j}(k)+2 \delta_{01}(k)\right]$ in the sine argurnent, turns out to be always negative $\left(R_{\max }<R_{, j}\right)$ and typically between $0.1 \AA$ and $0.5 \AA$. The shape of the peakin $R$ space is determined by the convolution of the structural Gaussian $N_{j} \exp \left[-\left(R-R_{j}\right)^{2} / 2 \sigma_{j}^{2}\right]$ with the Fourier transform of $\left\{\left|f_{j}(k, \pi)\right|\right.$ $\left.\times \exp \left[-2 R_{j} / \lambda(k)\right]\right\}$. Therefore in principle doing the analysis of the shape of FT peaks, one can get $R_{i j}, N_{j}$, and $\sigma_{j}^{2}$ for each coordination shell. However, for different reasons, generally the analysis in $R$ space is limited to low accurate distance determinations (typical accuracy $0.05-0.10 \AA$ ) and to only a qualitative view of the amplitude and width of the peaks. The main reason is the deformation introduced in the FT by the transformation $k$ range, whose lower limit $k_{\text {min }}$ cannot be reduced to zero, since below $2-3 \AA^{-1}$ the theoretical expression (15) is no more valid. To achieve the above quoted high accuracy, the analysis must be performed in $k$ space, by inverse Fourier transforming a given $R$ range back to $k$ space to isolate the contribution of one or more coordination shell, and then fitting the so Fourier filtered spectra with one or more sinusoidal contributions in accordance with Eq. (15). The free pararneters for each coordination shell are $R_{j}, N_{j}$, and $\sigma_{j}^{2}$ plus $E_{0}$. In such a way, the fitting procedure will change the $k$ scale, choosing the best value of $E_{0}$, i.e. the value which reduces the contribution of each coordination shell to a pure sinusoidal contribution.

\subsection{Some applications of EXAFS}

One of the main characteristics of EXAFS is its ability to detect the local structure of a system around an absorber, without requiring long range order. This characteristic favored the application of EXAFS to the study of disordered systems like metallic glasses, amorphous semiconductors, glasses and liquids, of dilute systems like clusters, impurities, surfaces and biological macromolecules, of multicomponent systems, like alloys. To give an idea of the power of the technique we now illustrate some applications. 


\subsubsection{Semiconductors alloys}

Pseudobinary serniconducting solid solutions of the $\mathrm{A}_{1-x} \mathrm{~B}_{x} \mathrm{C}$ (cation alloy) and $\mathrm{DB}_{x} \mathrm{~A}_{1-x}$ (anion alloy) are useful compounds for device applications as the band gap can be tuned with composition. For these semiconductors alloys spontaneous atomic scale ordering has been reported at specific compositions. Our current understanding of the phenomenon, however, is still low and a better knowledge of the microstructure in different growing conditions and compositions is needed. In fact, if ordering could be controlled and made to occur over large regions it would undoubtedly have the potential for providing materials for improved electronic and photonic devices.

X-ray diffraction measurements on pseudobinary alloys show that these materials have a zinc-blende structure, with one of the two fcc sublattices occupied by the $\mathrm{C}$ anion ( $\mathrm{D}$ cation) and the other occupied by both $\mathrm{B}$ and $\mathrm{A}$ cations (anions). With few exceptions, the lattice parameter $a(x)$ fulfills Vegard's law, i.e. it varies almost linearly between the values of the pure compounds $\mathrm{AC}$ and $\mathrm{BC}$ (DB and DA). It was surprising when EXAFS showed that bond lengths in the alloys have almost the same value as in the pure compounds, with nearest neighbor (NN) distances displaying a bimodal distribution [13-15] (Fig. 5). Moreover, all atoms of the chemically disordered sublattice occupy positions similar to the average lattice sites defined by the $\mathrm{X}$-ray lattice constant: this implies that the latter sublattice is much more distorted than the former.

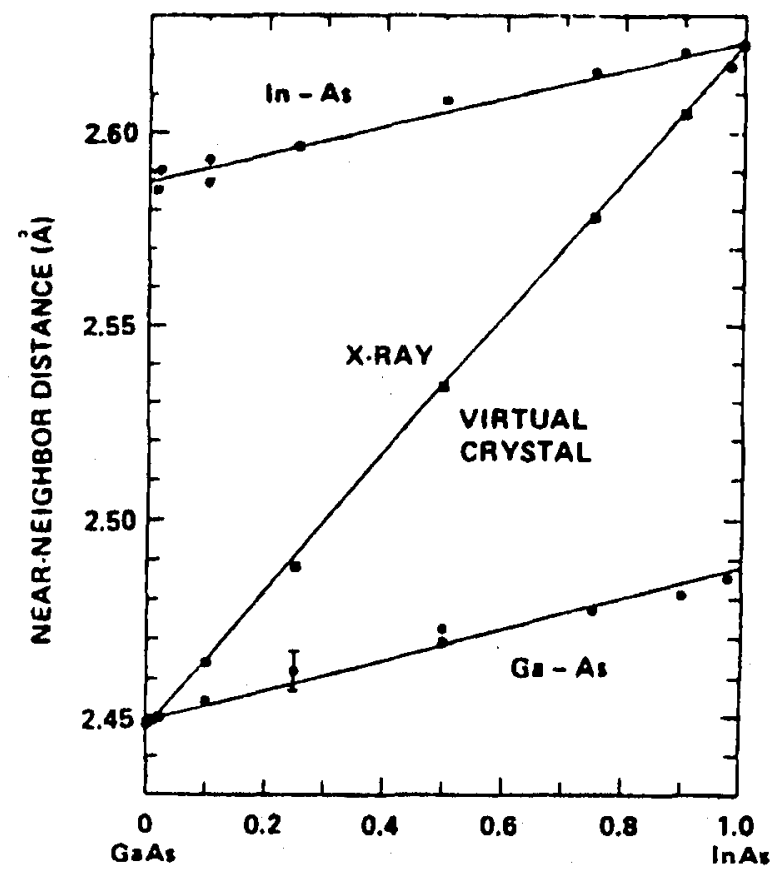

Fig. 5. Nearest neighbor distances in $\mathrm{Ga}_{1-x} \operatorname{In}_{x} \mathrm{As}$ alloys, showing the presence of bimodal distribution of distances $\mathrm{In}-\mathrm{As}$ and $\mathrm{Ga}-\mathrm{As}$. 
Today theoretical calculations have shown that the two bond lengths $R_{\mathrm{AC}}(x)$ and $R_{\mathrm{BC}}(x)$ can depend on alloy composition [16]. There are two limiting possibilities. From Bragg's and Pauling's notion that atomic radii are approximately conserved quantities and hence are transferable in different chemical environments, $R_{\mathrm{AC}}(x)$ and $R_{\mathrm{BC}}(x)$ are always composition independent and equal to the values in the pure compounds. In this case the bonds are completely relaxed to their covalent values, and the alloy is thought of as sustaining two chemically distinct bonds. On the other hand, due to Vegard's discovery, the distances in the alloy can be thought to be simple linear functions of the averaged lattice constant, in analogy with the distances in the pure solids. In this case the bonds are totally constrained by the host lattice, and the alloy is thought of as sustaining a single (average) chemical bond. EXAFS shows, however, that there is always a high degree of relaxation, the NN distances being always similar to the pure compound ones, showing only a small dependence on alloy composition.

A rather different behaviour is shown by the $\mathrm{Si}$ and $\mathrm{Ge}$ based amorphous alloys [17-20]. Figure 6 reports the NN bond lengths measured in a variety of amorphous semiconductor alloys as a function of composition (a-Si:H, a-Si $\mathrm{Si}_{1-x} \mathrm{Ge}_{x}: \mathrm{H}$, a-Si $\mathrm{Si}_{1-x} \mathrm{C}_{x}: \mathrm{H}, \quad a-\mathrm{Si}_{1-x} \mathrm{~N}_{x}: \mathrm{H}, \quad \mathrm{a}-\mathrm{Ge}_{1-x} \mathrm{~N}_{x}: \mathrm{H}$ and $\left.\mathrm{a}-\mathrm{Ge}_{1-x} \mathrm{Sn}_{x}: \mathrm{H}\right)$. The $\mathrm{NN}$ bond lengths are reported without identifying the particular alloy in which they are found; for example the $\mathrm{Si}-\mathrm{Si}$ distances shown are relative to a-Si:H, a-Si $\mathrm{Si}_{1-x} \mathrm{Ge}_{x}: \mathrm{H}$, a-Si $i_{1-x} \mathrm{C}_{x}: \mathrm{H}$ and $\mathrm{a}-\mathrm{Si}_{1-x} \mathrm{~N}_{x}: \mathrm{H}$.

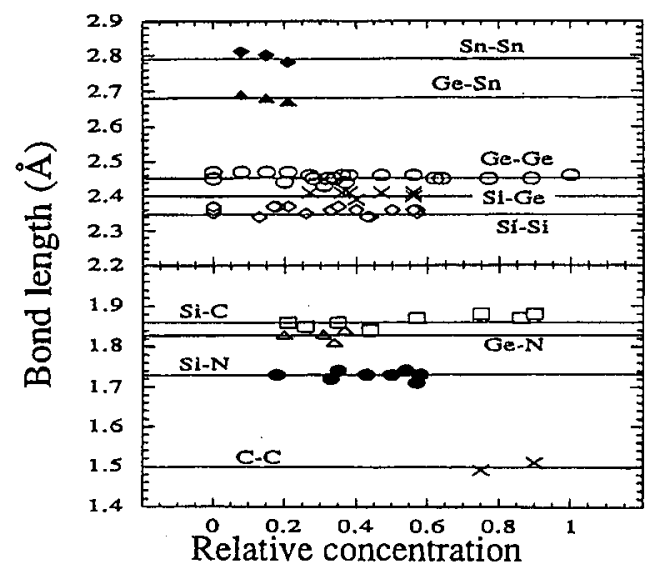

Fig. 6. Nearest neighbor distances in amorphous semiconductor alloys.

Two important conclusions can be drawn from the figure:

a) within the overall experimental uncertainty of $0.03 \AA$ there is no variation in the NN bond lengths with composition within the same alloy;

b) no differences in the same bond length are found between the various alloys studied.

The NN bond length is a conserved quantity, equal to the value determined by the NN interactions and independent of the surrounding matrix. While in crys- 
talline semiconductor alloys a weak variation in bond lengths remains, this is totally absent in amorphous alloys due to the absence of the constraint of long range order.

The chemical composition of the first shell is also of great importance. XAFS directly measures coordination numbers so that by comparing this quantity with the average atomic concentration it is possible to distinguish between various degrees of chemical ordering. Extreme cases for an alloy of composilion $\mathrm{A}_{1-x} \mathrm{~B}_{x}$ are represented by clustering (tendency against $A-B$ bonds), random distribution and chemical ordering (tendency for $\mathrm{A}-\mathrm{B}$ bonds). By comparing the degree of chemical ordering in different systems it was possible to draw some general conclusions on what determines the degree of chemical ordering in amorphous semiconductor alloys. Figure 7 shows, as an example, the chemical order analysis for the technologically important alloy a-Si $\mathrm{S}_{1-x} \mathrm{C}_{x}: \mathrm{H}$; the ratio of $\mathrm{Si}-\mathrm{C}$ bonds to the total $\mathrm{Si}$ coordination as a function of $\mathrm{C}$ concentration is reported. Also reported in the figure are the expected behaviors for a random distribution and for total chemical ordering. It is quite clear from the figure that there is a strong tendency for chemical ordering.

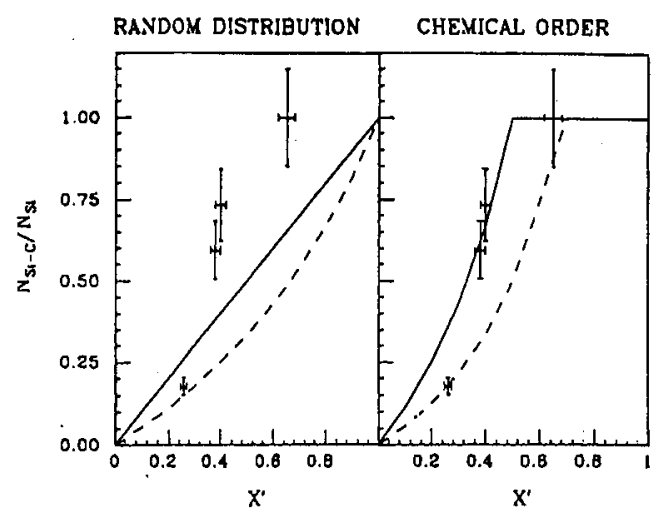

Fig. 7. Experimental fractional coordination number of carbon atoms around silicon, compared with different ordering hypothesis.

In a recent theoretical approach towards the problern of chemical ordering in amorphous semiconductor alloys [21] it has been found that minimization of the free energy leads to a nearly complete chemical ordering whenever there is a large electronegativity difference between alloying elements (i.e. when heteroatomic bonds are stronger than homoatomic ones). The lack of long range order does not lead, in these cases, to a random chemical arrangement. The EXAFS results completely agree with these predictions.

\subsubsection{Metallic clusters}

Microclusters represent a special state of matter, they are aggregates of atoms too large to be called molecules but still too small to have the structure of a crystal and can therefore provide information about the transition from the molecular to the bulk state. In addition, microclusters can supply convenient theoretical 
and experimental models for chemisorption, nucleation, photographic emulsions, and catalysis.

Many studies have been and are being performed on the electronic and structural properties of microclusters under different conditions such as different chemical environment, sample preparation, size, and substrates. Many problems are still unsolved: for instance, it is unknown if the cluster-size-dependent chemical reactivity is due to a change in their electronic or in their structural properties and how these properties depend on the sample preparation method and on the interaction with substrates.

Clusters are an ideal systern to study with XAS, since they form a dilute system without long range order. Figure 8 shows the nearest-neighbor distances as a function of the cluster dimension obtained by EXAFS for Au cluster [22]. A contraction is observed, which reaches a value of $2.5 \%$ for the $11 \AA$ clusters. Such behavior is fully explained by a simple macroscopic liquid-drop model: clusters are imagined as homogeneous spheres on which isotropic surface stress acts, shortening the lattice parameter with respect to the bulk one [23].

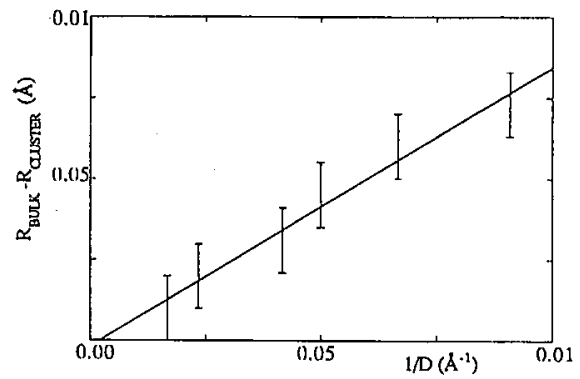

Fig. 8. Plot of nearest-neighbor distance contraction versus the inverse of the cluster mean diameter. The straight line is a linear fit to the data.

A still open question is the presence at small cluster dimension of a structural phase transition from the fcc structure to an icosahedral one. Such phase transition is forseen by theoretical calculations on the cluster stability, but was never experimentally observed. In the icosahedra the first fcc shell is split into two shells centered at distances $R_{\mathrm{I}}$ and $R_{\mathrm{II}}$ and related by $R_{\mathrm{I}}=1.05 R_{\mathrm{II}}$. It is possible to detect the presence of icosahedra performing fits of the inverse Fourier transform spectra of the NN Fourier transform peak, using a fcc model and an icosahedral model. In the case of Au, only using a single-shell fcc model good fits were obtained for all samples. The question of whether the structure is still fcc up to the second and third shells can be fully clarified also from the XANES spectra, which have a typical fcc shape in going from the bulk to the $11 \AA$ clusters (Fig. 9). It can be seen that as the cluster size decreases there is a shift to higher energies and a broadening of the XANES peaks. The former is due to the shortening of the lattice parameters, while the latter is a consequence of both the cluster-size distribution inside each sample and of the different weight of the second and third shell contributions to the spectra of the different samples. 


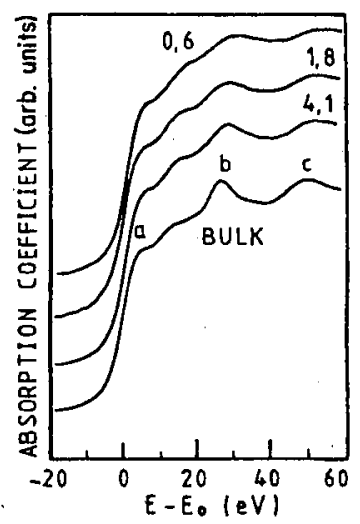

Fig. 9. XANES spectra of gold clusters for different cluster dimensions.

\subsubsection{Metallic glasses}

The glassy state is not a stable configuration for matter. Actually a glass is an undercooled liquid that continously evolves towards the equilibrium configuration at room temperature that is the crystalline state. In the case of metallic glasses, whose melting temperatures range between 600 and $1000^{\circ} \mathrm{C}$, we have relatively stable materials down $200^{\circ} \mathrm{C}$. This intrinsic instability is the main drawback of these materials that can seriously compromise their technological application. The crystallization of a glass is an usually temperature induced process, during which the material evolves to the ordered state. It is a first order phase transition, with a rather high enthalpy, initiated by small crystalline nuclei in the matrix. The nuclei can be "frozen" crystallites present in the melt or self-nucleating in the solid material. However formed the nuclei, their growth is controlled by the jump of atoms from the amorphous matrix in the crystallite governed by the Arrhenius law and by the diffusion of the atoms towards (or away from) the crystallite border. All those different crystallization processes leave a footprint in the time evolution of the crystallized fraction $x$. Following Mehl-Avrami the time evolution $x(t)$ in an isothermal process is given by

$$
x(t)=1-\exp \left(-b t^{n}\right)
$$

where the pararneter $n$ takes different values in the different cases. In this way a careful determination of $n$ can reveal the physical processes that drive the crystallization.

An EXAFS study [24] of the reordering of a NiB amorphous alloy during an isothermal annealing is shown in Fig. 10. The increase in the first peak of the radial distribution function is the main effect of the temperature-induced reordering. A complete analysis of the EXAFS data shows that in this simple case the crystallization process consists of a continuous growing of a $\mathrm{Ni}-\mathrm{fcc}$ ordered matrix in the glass without appreciable contribution of other phases. The ordered matrix resulted to be formed by nanocrystals with an interatomic spacing lower than that in the macroscopic fcc crystals and a reduced number of nearest. neighbors. These facts were explained postulating a large (30\%) substitutional B doping in the metal 


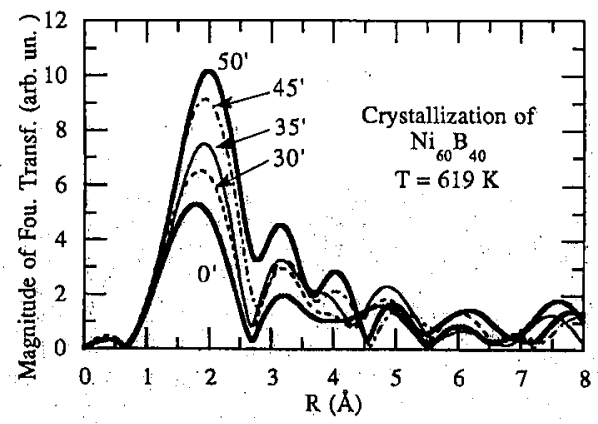

Fig. 10. Isothermal crystallization spectra of $\mathrm{Ni}_{60} \mathrm{~B}_{40}$.

matrix. A deeper insight in the temporal behavior of the structural parameters showed that the main effect of the reordering is the sharpening of the distribution of the first neighbors without changing their nuraber or distance. The EXAFS data allowed also a description from a kinetical point of view. The crystallization resulted to be a simple growth of nuclei driven by interface processes. Evidently the small size of the crystallites played a central role in frustrating the diffusion processes that usually drive the segregation of ordered metallic matrices.

\section{Multiple scattering}

To show an example of the relevance of the multiple scattering paths in analyzing XAS spectra, we will illustrate now the pioneering results obtained year's ago by the Frascati group in the case of $\left(\mathrm{MnO}_{4}\right)^{-}$and $\left[\mathrm{Mn}\left(\mathrm{H}_{2} \mathrm{O}_{6}\right)\right]^{2+}$ ions in soIution [25]. In the first case the $\mathrm{Mn}$ atoms are tetrahedrally surrounded by the oxygen, while in the second the coordination geometry is octahedral. The study of such cornplexes in aqueous solution ensures the absence of EXAFS second shell structural signal inside the $\mathrm{Mn}-\mathrm{O}$ cluster, which generally can hinder the multiple scattering contributions. Figure 11a shows the comparison of the experimental spectra with the result of full multiple scattering calculations. Figure $11 \mathrm{~b}$ and 11c show the breakdown in partial contribution. Above $40 \mathrm{eV}$ the surn of the atomic-like contribution and the EXAFS one, well reproduces the spectrum. Below $40 \mathrm{eV}$, sizeable contribution from triple and quadruple scattering are revealed. Therefore it would seem that the EXAFS regime starts at this energy. This is due to a fortuitous cancellation of the triple and quadruple contributions, which are comparable in magnitude with the EXAFS in the whole energy range, but opposite in phase one with each other. At energies below $20 \mathrm{eV}$, the series of multiple scattering contribution does not converge, so its sum cannot reproduce the spectrum, while the full multiple scattering calculation does. The situation is quite different for the $\left(\mathrm{MnO}_{4}\right)^{-}$complex (Fig. 11d,e,f). In this case in the energy above $40 \mathrm{eV}$ the surn of the atomic-like contribution, the EXAFS and the triple scattering reproduces the experimental spectrum, the quadruple one being negligible. Therefore the triple scattering must be considered in analyzing the spectra. At lower energies also higher order contributions must be considered, to reproduce the spectral 

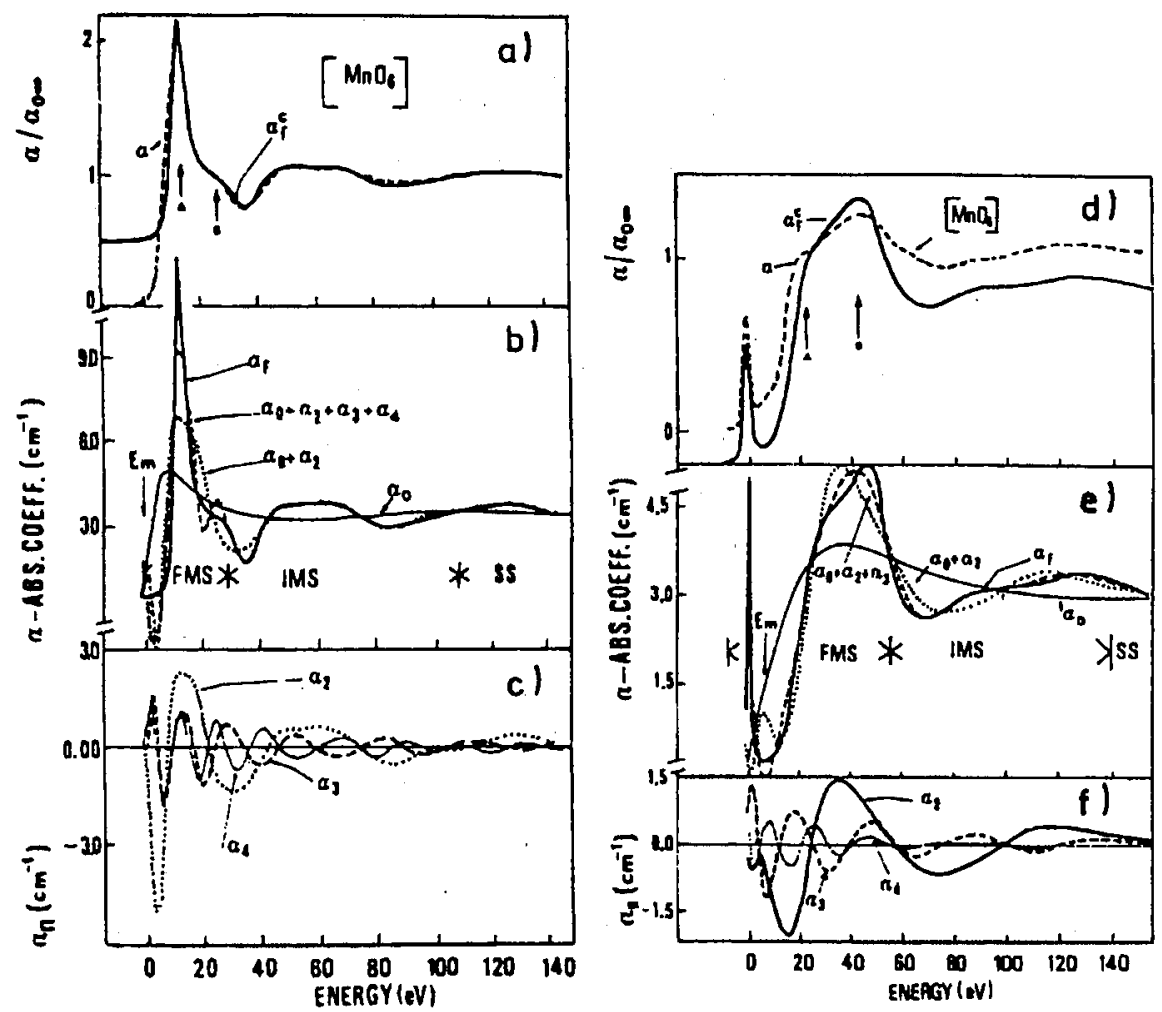

Fig. 11. Comparison between experimental and calculated spectra of $\mathrm{Mn}\left(\mathrm{H}_{2} \mathrm{O}_{6}\right)^{2+}$ $(\mathrm{a}, \mathrm{b}, \mathrm{c})$ and $\left(\mathrm{MnO}_{4}\right)^{-}(\mathrm{de}, \mathrm{f})$ complexes. $\alpha_{j}$ is the single contribution to the spectra due to scattering path of $j$-th order.

features $\mathrm{A}$ and $\mathrm{B}$. The breakdown approach in terms of partial contributions to the absorption coefficient shows the continuous merging of the multiple scattering regime into the EXAFS regime. The merging interval is much larger than generally believed, since can reach up to $150 \mathrm{eV}$. So often, the analysis of a XAS spectrum in term of EXAFS contribution only neglects sizeable contribution from the multiple scattering paths. Data analysis procedures to account for this contributions have been successfully applied in many cases, so now a new structural probe is available even if its use is still in the hand of few groups in the world.

An exciting result was obtained using the above-mentioned breakdown approach in terms of partial contributions to the absorption coefficient in amorphous hydrogenated Si [26], where next near neighbor EXAFS contribution as well as multiple scattering were determined. In this case the spectra are dominated by the contribution of the first neighbor EXAFS, as usually in disordered materials. Using an original and sophisticated procedure [26], this contribution was determined and then subtracted from the experimental spectra. After that, in the spectra a small residual signal is left due to multiple scattering and EXAFS of next 


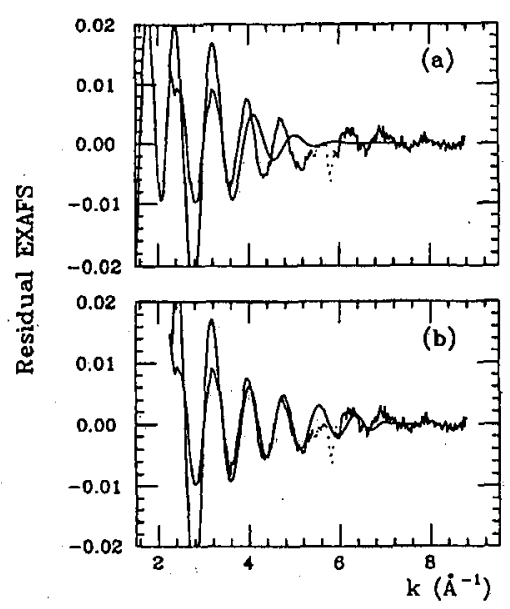

Fig. 12. Comparison between the experimental high frequency residual of a-Si sample with: (a) single scattering contributions associated with the second peak in $g_{2}$; (b) the sum of all single and multiple scattering contributions coming from $g_{2}$ and $g_{3}$.

neighbor shells (Fig. 12). If one uses any model for the structure of a-Si and builds up a theoretical second shell EXAFS signal as the average of all EXAFS signals of the second neighbors possible pairs present in the model, one discovers that a large phase mismatch is present between theory and experiment (Fig. 12a). This phase mismatch is largely accounted for if one adds to the theory also the average of the triple contribution, calculated using the third term of the multiple scattering series expansion (Fig. 12b). This result clearly shows the presence of MS signals in the a-Si spectra, and therefore that XAS spectra can be used to determine or at least to check theoretical evaluation of the three-body correlation function in many systems.

\section{References}

[1] E.A. Stern, Phys. Rev. B 10, 3027 (1974).

[2] P.A. Lee, J.B. Pendry, Phys. Rev. B 11, 2795 (1975).

[3] D.E. Sayers, F.W. Lytle, E.A. Stern, Phys. Rev. Lett. 27, 1204 (1971).

[4] P.A. Lee, P.H. Citrin, P. Eisenberger, B.M. Kincaid, Rev. Mod. Phys. 53, 769 (1981).

[5] T.M. Hayes, J.B. Boyce, Solid State Phys. 37, 173 (1981).

[6] X-Ray Absorption, Eds. D.C. Koningsberger, R. Prins, Wiley, New York 1988.

[7] C.R. Natoli, M. Benfatto, S. Doniach, Phys. Rev. A 34, 4682 (1986).

[8] W.L. Schaich, Phys. Rev. B 8, 4028 (1973).

[9] B.K. Teo, P.A. Lee, J. Am. Chem. Soc. 101, 2815 (1979).

[10] A.G. McKale, B.W. Veal, A.P. Paulikas, S.K. Chan, G.S. Knapp, J. Am. Chem. Soc. 110, 3763 (1988).

[11] E.A. Stern, B.A. Bunker, S.M. Heald, Phys. Rev. B 21, 5521 (1980). 
[12] See for example, J. Mustre de Leon, J.J. Rehr, I.I. Zabinsky, Phys. Rev. B 44, 4146 (1991).

[13] J.C. Mikkelsen Jr., J.B. Boyce, Phys. Rev. Lett. 49, 1412 (1982).

[14] A. Balzarotti, M. Czyzyk, A. Kisiel, N. Motta, M. Podgorny, M. Zimnal-Starnawska, Phys. Rev. B 30, 2295 (1984).

[15] A. Balzarotti, M. Czyzyk, A. Kisiel, N. Motta, M. Podgorny, M. Zimnal-Starnawska, Phys. Rev. B 31, 7526 (1985).

[16] Y. Cai, M.F. Thorpe, Phys. Rev: B 46, 15872 (1992):

[17] L. Incoccia, S. Mobilio, M.G. Proietti, P. Fiorini, C. Giovannella, F. Evangelisti, Phys. Rev. B 31, 1028 (1985).

[18] S. Pascarelli, F. Boscherini, S. Mobilio; F. Evangelisti, Phys. Rev. B 45, 1650 (1992).

[19] F. Boscherini, A. Filipponi, S. Pascarelli, F. Evangelisti, S. Mobilio, F.C. Marques, I. Chambouleyron, Phys. Rev. B 39, 8364 (1989).

[20] S. Pascarelli, F. Boscherini, S. Mobilio, A.R. Zanatta, F.C. Marques, I: Chambouleyron, Phys. Rev. B 46, 6718 (1992).

[21] Z. Yin, F.W. Smith, Phys. Rev. B 43, 4507 (1991).

[22] A. Balerna, E. Bernieri, P. Picozzi, A. Reale, S. Santucci, E. Burattini, S. Mobilio, Phys. Rev. B 31, 5058 (1985).

[23] C.W. Mays, J.S. Vermaak, D. Kuhlmann-Wilsorf, Surf. Sci. 12, 134 (1968).

[24] F. d'Acapito, F. Buffa, G. Vlaic, F. Boscherini, S. Mobilio, J. Non-Crystalline Solids 156, 571 (1993).

[25] M. Benfatto, C.R. Natoli, A. Bianconi, J. Garcia, A. Marcelli, M. Fanfoni, I. Davoli, Phys. Rev. B 34, 5774 (1986).

[26] A. Filipponi, F. Evangelisti, M. Benfatto, S. Mobilio, C.R. Natoli, Phys. Rev. B 40, 9636 (1989). 